\title{
Geometric Defuzzification Revisited
}

\author{
Sarah Greenfield ${ }^{\mathrm{a}, *}$ \\ ${ }^{a}$ DMU Interdisciplinary Group in Intelligent Transport Systems (DIGITS), \\ School of Computer Science and Informatics, \\ De Montfort University, Leicester LE1 9BH, UK.
}

\begin{abstract}
In this paper the Geometric Defuzzification strategy for type-2 fuzzy sets is reappraised. For both discretised and geometric fuzzy sets the techniques for type-1, interval type-2, and generalised type- 2 defuzzification are presented in turn. In the type- 2 case the accuracy of Geometric Defuzzification is assessed through a series of test runs on interval type-2 fuzzy sets, using Exhaustive Defuzzification as the benchmark method. These experiments demonstrate the Geometric Defuzzifier to be wildly inaccurate. The test sets take many shapes; they are not confined to those type- 2 sets with rotational symmetry that have previously been acknowledged by the technique's developers to be problematic as regards accuracy. Type2 Geometric Defuzzification is then examined theoretically. The defuzzification strategy is demonstrated to be built upon a fallacious application of the concept of centroid. This explains the markedly inaccurate experimental results. Thus the accuracy issues of type-2 Geometric Defuzzification are revealed to be inevitable, fundamental and significant.
\end{abstract}

Keywords: Geometric Type-2 FIS, Geometric Defuzzification, Type-2 Defuzzification, Interval Type-2 Fuzzy Set, Centroid, Fallacy

\section{Introduction}

This paper is a critique of the technique of Geometric Defuzzification for type2 fuzzy sets. Fuzzy set theory, and its correlate, fuzzy logic, were originated by Lotfi Zadeh [26] in the 1960s. Fuzzy logic is implemented in software through the Fuzzy Inferencing System (FIS), which may be of any type. The type of the

\footnotetext{
${ }^{*}$ Corresponding author

Email address: s.greenfield@dmu.ac.uk (Sarah Greenfield)
} 
FIS is determined by the highest type of the fuzzy sets employed within it. There are two sorts of type-2 FISs: 1. The Mamdani FIS for which the membership function output is a type-2 fuzzy set that requires defuzzification; 2 . The TakagiSugeno-Kang FIS, with output membership functions that are either constant or linear; defuzzification is therefore unnecessary. This paper, following the work on Geometric Defuzzification, is concerned solely with the Mamdani style FIS.

A Mamdani FIS (of any type) consists of three stages: fuzzification, inferencing, and defuzzification:

1. Fuzzification is the process in which a crisp input value's degree of membership of a fuzzy set is determined, based on the membership function of the fuzzy set.

2. Inferencing, which may be further subdivided into three stages: (a) Antecedent computation; (b) Implication; (c) Aggregation. The fuzzy set output by this stage is termed the aggregated set.

3. Defuzzification, in which the aggregated set is transformed into a crisp number, the output of the FIS.

The Geometric Defuzzification technique may be applied unproblematically to type-1 fuzzy sets [2].

Type-2 defuzzification conventionally breaks down into two stages [23] (Figure 1):

1. Type-reduction, which transforms the type-2 fuzzy set into a type-1 fuzzy set;

2. Type-1 defuzzification of the resultant fuzzy set, converting it into a crisp number.

Type-reduction in the mathematically justified form of Exhaustive Defuzzification [11] (Subsection 3.1) is notoriously computationally complex, inspiring researchers to develop alternative approximate strategies [10-12, 16-19], among them Coupland and John, the authors of the Geometric Defuzzifier. They express the motivation for their innovation thus [6, page 929]:

"DEFUZZIFICATION is a critical stage in any Mamdani fuzzy inference system (FIS). It needs to be simple enough to permit system execution in real time on embedded hardware and yet must be precise and accurate so that correct decisions are taken." 


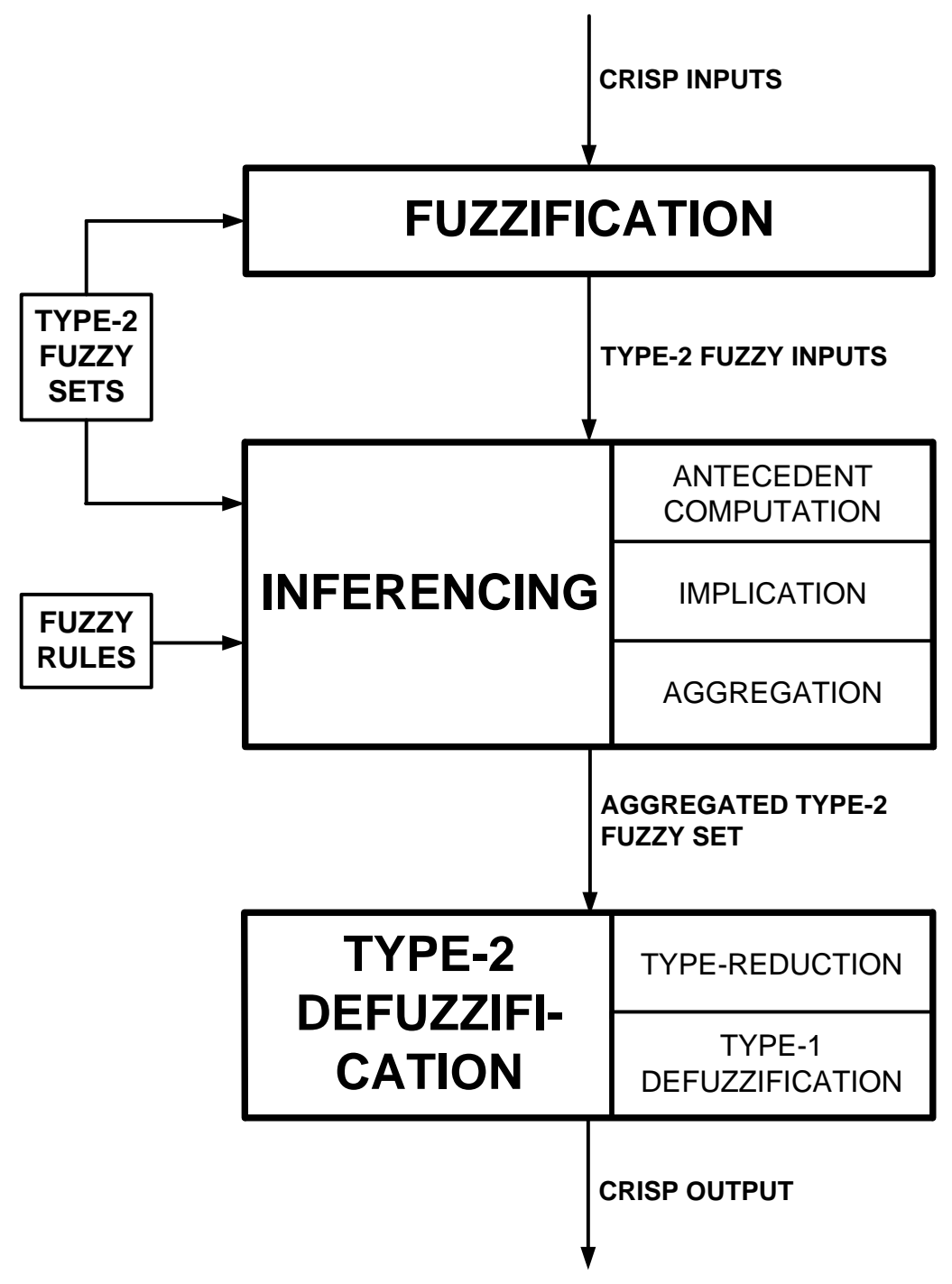

Figure 1: The Mamdani Type-2 FIS [13]. 
The type-2 Geometric Defuzzifier is appealingly simple as it totally eliminates the stage of type-reduction. Dating from 2005, when the interval version was presented [4], the method has been published since in [6-8] in both its interval and generalised versions. In the context of the Geometric FIS, the technique has been presented in $[2,3,5]$.

That this technique performs unreliably with certain forms of type-2 fuzzy set was acknowledged by its developers in 2008:

"The only known form of a set that is problematic for the Geometric

Defuzzifier is one that has rotational symmetry." [6, page 940]

This caveat was reiterated in 2013 [7, page 95].

The purposes of the present paper are:

- To show that the type-2 Geometric Defuzzifier's accuracy issues are pervasive and endemic across type- 2 sets of all shapes, not just those that are rotationally symmetrical;

- To reveal the flawed reasoning underlying type-2 Geometric Defuzzification.

Geometric fuzzy sets are presented as differing conceptually from discretised fuzzy sets $[2,5]$, but it is disputable whether the distinction is more than superficial. At any rate the definitions of geometric and discretised fuzzy sets permit creation of type-1 and type-2 fuzzy sets that may be construed as either geometric or discretised. The interval type-2 fuzzy sets deployed as examples below are of this dual nature.

The structure of the remainder of this paper is as follows: The next section deals with background, definitions and assumptions relevant to the rest of the paper, after which, in Section 2, the defuzzification of type-1 fuzzy sets is discussed. Following that, Section 3 explores type-2 defuzzification. In Section 4, the Geometric Defuzzifier is experimentally evaluated in relation to the defuzzification of interval type- 2 fuzzy sets. In Section 5 the fundamental flaw with type- 2 Geometric Defuzzification is revealed. Section 6 concludes the paper, offering suggestions for further research.

\subsection{Preliminaries}

\subsubsection{Fuzzy Sets: Definitions}

"Let $X$ be a universe of discourse. A type-1 fuzzy set $A$ on $X$ is characterised by a membership function $\mu_{A}: X \rightarrow[0,1]$ and can be expressed as follows [26]:

$$
A=\left\{\left(x, \mu_{A}(x)\right) \mid \mu_{A}(x) \in[0,1] \forall x \in X\right\} .
$$


Note that the membership grades of $A$ are crisp numbers. In the following we will use the notation $U=[0,1] . "[12]$

"Let $\tilde{P}(U)$ be the set of fuzzy sets in $U$. A type-2 fuzzy set $\tilde{A}$ in $X$ is a fuzzy set whose membership grades are themselves fuzzy [27-29]. This implies that $\mu_{\tilde{A}}(x)$ is a fuzzy set in $U$ for all $x$, i.e. $\mu_{\tilde{A}}: X \rightarrow \tilde{P}(U)$ and

$$
\tilde{A}=\left\{\left(x, \mu_{\tilde{A}}(x)\right) \mid \mu_{\tilde{A}}(x) \in \tilde{P}(U) \forall x \in X\right\} .
$$

It follows that $\forall x \in X \exists J_{x} \subseteq U$ such that $\mu_{\tilde{A}}(x): J_{x} \rightarrow U$. Applying (1), we obtain:

$$
\mu_{\tilde{A}}(x)=\left\{\left(u, \mu_{\tilde{A}}(x)(u)\right) \mid \mu_{\tilde{A}}(x)(u) \in U \forall u \in J_{x} \subseteq U\right\} .
$$

$X$ is called the primary domain and $J_{x}$ the primary membership of $x$ while $U$ is known as the secondary domain and $\mu_{\tilde{A}}(x)$ the secondary membership of $x$.

Putting (2) and (3) together we obtain

$$
\tilde{A}=\left\{\left(x,\left(u, \mu_{\tilde{A}}(x)(u)\right)\right) \mid \mu_{\tilde{A}}(x)(u) \in U, \forall x \in X \wedge \forall u \in J_{x} \subseteq U\right\} . "[15]
$$

Definition 1 (Interval Type-2 Fuzzy Set [14]). "An interval type-2 fuzzy set is a type-2 fuzzy set whose secondary membership grades are all 1.”

For interval type-2 fuzzy sets, Equation 4 reduces to:

$$
\tilde{A}=\left\{(x,(u, 1)), \forall x \in X \wedge \forall u \in J_{x} \subseteq U\right\} .
$$

Definition 2 (Embedded Set [14]). "Let $\tilde{A}$ be a type-2 fuzzy set in X. For discrete universes of discourse $X$ and $U$, an embedded type- 2 set $\tilde{A}_{e}$ of $\tilde{A}$ is defined as the following type-2 fuzzy set

$$
\tilde{A}_{e}=\left\{\left(x_{i},\left(u_{i}, \mu_{\tilde{A}}\left(x_{i}\right)\left(u_{i}\right)\right)\right) \mid \forall i \in\{1, \ldots, N\}: x_{i} \in X u_{i} \in J_{x_{i}} \subseteq U\right\} .
$$

$\tilde{A}_{e}$ contains exactly one element from $J_{x_{1}}, J_{x_{2}}, \ldots, J_{x_{N}}$, namely $u_{1}, u_{2}, \ldots, u_{N}$, each with its associated secondary grade, namely $\mu_{\tilde{A}}\left(x_{1}\right)\left(u_{1}\right), \mu_{\tilde{A}}\left(x_{2}\right)\left(u_{2}\right), \ldots$, $\mu_{\tilde{A}}\left(x_{N}\right)\left(u_{N}\right)$."

Definition 3 (Slice [14]). "A slice of a type-2 fuzzy set is a plane either

1. through the $x$-axis, parallel to the $u$-z plane, or

2. through the u-axis, parallel to the $x$-z plane."

Definition 4 (Vertical Slice [14, 24]). "A vertical slice of a type-2 fuzzy set is a plane through the $x$-axis, parallel to the u-z plane." 
Definition 5 (Horizontal Slice). A horizonal slice of a type-2 fuzzy set is a plane through the u-axis, parallel to the $x$-z plane.

Definition 6 (Footprint Of Uncertainty [12]). "FOU stands for Footprint Of Uncertainty, the projection of the type-2 fuzzy set onto the $x$-u plane."

Definition 7 (Lower Membership Function [12]). "The Lower Membership Function (LMF) of a type-2 fuzzy set is the type-1 membership function associated with the lower bound of the FOU."

Definition 8 (Upper Membership Function [12]). “The Upper Membership Function (UMF) of a type-2 fuzzy set is the type-1 membership function associated with the upper bound of the FOU."

Definition 9 (Vertical Symmetry). A fuzzy set has vertical symmetry if it has reflectional symmetry in a vertical slice.

Definition 10 (Horizontal Symmetry). A fuzzy set has horizontal symmetry if it has reflectional symmetry in a horizontal slice.

Definition 11 (Degree of Discretisation [14]). "The degree of discretisation is the separation of the slices."

For a type-2 fuzzy set discretisation is applied to both the primary and secondary domains. The primary domain is discretised into vertical slices. The primary and secondary domains do not necessarily have the same degree of discretisation and that of the secondary domain may vary from one vertical slice to another.

\subsubsection{Geometric Fuzzy Sets: Definitions}

Definition 12 (Geometric Type-1 Fuzzy Set [7]). "A geometric type-1 fuzzy set is a series of ordered vertices that are connected by line segments to form a function over a continuous domain. This function is linear in all but a finite set of points. A geometric fuzzy set $A$ over the domain $X$ consists of pairs of vertices $(x, y)$ where the $x \in X$ and the $y$ components of all the vertices are in the interval $[0,1]$ i.e.,

$$
\mu_{A}: X \rightarrow[0,1] . ”
$$

Definition 13 (Generalised Geometric Type-2 Fuzzy Set [7]). "A geometric type2 fuzzy set is defined as a collection of $n$ triangles in $3 D$ space where the edges of these triangles connect to form a $3 D$ polyhedron, i.e.

$$
\tilde{A}=\bigcup_{i=1 \ldots n} t^{i} \text { where } t^{i}=\left[\begin{array}{ccc}
x_{1}^{i} & y_{1}^{i} & z_{1}^{i} \\
x_{2}^{i} & y_{2}^{i} & z_{2}^{i} \\
x_{3}^{i} & y_{3}^{i} & z_{3}^{i}
\end{array}\right]
$$


where $x_{1}^{i}, x_{2}^{i}$ and $x_{3}^{i} \in X$ and $y_{1}^{i}, y_{2}^{i}, y_{3}^{i}, z_{1}^{i}, z_{2}^{i}$ and $z_{3}^{i} \in[0,1]$."

Definition 13 is readily adapted to the interval geometric type-2 fuzzy set:

Definition 14 (Interval Geometric Type-2 Fuzzy Set). An interval geometric type2 fuzzy set is defined as a collection of n triangles in $3 D$ space where the edges of these triangles connect to form a $3 D$ polyhedron, i.e.

$$
\tilde{A}=\bigcup_{i=1 \ldots n} t^{i} \text { where } t^{i}=\left[\begin{array}{lll}
x_{1}^{i} & y_{1}^{i} & 1 \\
x_{2}^{i} & y_{2}^{i} & 1 \\
x_{3}^{i} & y_{3}^{i} & 1
\end{array}\right]
$$

where $x_{1}^{i}, x_{2}^{i}$ and $x_{3}^{i} \in X$ and $y_{1}^{i}, y_{2}^{i}$ and $y_{3}^{i} \in[0,1]$.

As with the discretised interval type- 2 fuzzy set, the geometric interval type-2 fuzzy set is completely defined by its FOU.

\subsubsection{Assumptions}

1. It is assumed, without loss of generality, that a type-2 fuzzy set is contained in the unit cube and may be regarded as a set of points of the form $(x, u, z)$.

2. The centroid method of type-1 defuzzification is employed throughout the research reported below.

3. Exhaustive Defuzzification is taken to be the standard for accuracy for the defuzzification of type-2 fuzzy sets.

4. The minimum t-norm is used in Exhaustive Defuzzification.

The next section concerns the defuzzification of discretised and geometric type-1 fuzzy sets.

\section{Defuzzification of Type-1 Fuzzy Sets}

\subsection{Conventional Type-1 Defuzzification}

Definition 15 (Centroid of a Discretised Type-1 Fuzzy Set). "Let A be a nonempty type-1 fuzzy set discretised into $m$ vertical slices at $x_{1}, x_{2}, \ldots, x_{m}$. The centroid of $A$ is calculated by this formula:

$$
X_{A}=\frac{\sum_{i=1}^{i=m} \mu_{A}\left(x_{i}\right) x_{i}}{\sum_{i=1}^{i=m} \mu_{A}\left(x_{i}\right)} .
$$




\subsection{Geometric Type-1 Defuzzification}

To defuzzify a geometric type-1 fuzzy set, its membership function is converted into a closed polygon by the inclusion of points on the $x$-axis, and if necessary points on $x=0$ and $x=1$ [2].

Definition 16 (Centroid of a Geometric Type-1 Fuzzy Set [5]). "Let A be a nonempty geometric type-1 fuzzy set augmented to form a closed polygon, consisting of $n$ vertices $\left(x_{1}, y_{1}\right),\left(x_{2}, y_{2}\right), \ldots,\left(x_{n}, y_{n}\right)$. The centroid of $A$ is calculated by this formula:

$$
X_{A}=\frac{\sum_{i=1}^{i=n}\left(x_{i}+x_{i+1}\right)\left(x_{i} y_{i+1}-x_{i+1} y_{i}\right)}{3 \sum_{i=1}^{i=n}\left(x_{i} y_{i+1}-x_{i+1} y_{i}\right)} .
$$

The formulae in Definitions 15 and 16 are not equivalent but produce good approximations to each other.

\section{Defuzzification of Type-2 Fuzzy Sets}

\subsection{Exhaustive Type-Reduction}

For type-1 fuzzy sets defuzzification is computationally simple, with a choice of several strategies, among them the centroid and the centre of maxima [21]. Type-2 defuzzification is conventionally performed in two stages [23] ${ }^{1}$ :

1. Type-reduction, converting the type-2 fuzzy set to a type-1 fuzzy set known as the Type-Reduced Set (TRS);

2. Defuzzification of the resultant type-1 fuzzy set.

The TRS is defined thus:

Definition 17 (TRS of a Type-2 Fuzzy Set [14]). “The TRS associated with a type2 fuzzy set $\tilde{A}$ with primary domain $X$ discretised into $N$ points $X=\left\{x_{1}, x_{2}, \ldots, x_{N}\right\}$,

\footnotetext{
${ }^{1}$ This algorithm is derived from Zadeh's Extension Principle [27], which generalises operations defined on crisp numbers to type-1 fuzzy sets.
} 
is

$$
\begin{array}{r}
C_{\tilde{A}}=\left\{\left(\frac{\sum_{i=1}^{N} x_{i} \cdot u_{k_{i}}}{\sum_{i=1}^{N} u_{k_{i}}}, \mu_{\tilde{A}}\left(x_{1}\right)\left(u_{k_{1}}\right) * \ldots\right.\right. \\
\left.\ldots * \mu_{\tilde{A}}\left(x_{N}\right)\left(u_{k_{N}}\right)\right) \mid \forall\left(u_{k_{1}}, u_{k_{2}}, \ldots, u_{k_{N}}\right) \\
\left.\in J_{x_{1}} \times J_{x_{2}} \times \ldots \times J_{x_{N}} \subseteq U^{N}\right\},
\end{array}
$$

where * is a t-norm."

Based on Definition 17, Mendel and John's Representation Theorem [24] provides a straightforward, precise, type-reduction technique, which when presented in algorithmic form [12] makes reference to embedded sets (Definition 2). This algorithm has come to be known as the Exhaustive Method, as it necessitates each type-1 embedded set to be defuzzified [17]. The defuzzified value is then paired with the embedded set's minimum secondary membership grade. The set of ordered pairs constitutes the TRS.

Inevitably, discretisation brings approximation with it, but the mathematically rigourous Exhaustive Method does not engender inaccuracies beyond those produced by discretisation itself, hence its use as the standard for accuracy comparisons $[11,12]$. A significant drawback of the Exhaustive Method is its high computational complexity.

For the interval TRS Definition 17 reduces to:

Definition 18 (TRS of an Interval Type-2 Set [12]). "The TRS associated with an interval type-2 fuzzy set $\tilde{A}$ with primary domain $X$ discretised into $N$ points $X=\left\{x_{1}, x_{2}, \ldots, x_{N}\right\}$, is

$$
\begin{gathered}
C_{\tilde{A}}=\left\{\left(\frac{\sum_{i=1}^{N} x_{i} \cdot u_{k_{i}}}{\sum_{i=1}^{N} u_{k_{i}}}, 1\right) \mid \forall\left(u_{k_{1}}, u_{k_{2}}, \ldots\right.\right. \\
\left.\left.\ldots, u_{k_{N}}\right) \in J_{x_{1}} \times J_{x_{2}} \times \ldots \times J_{x_{N}} \subseteq U^{N}\right\} .
\end{gathered}
$$

\subsection{Geometric Defuzzification of Interval Type-2 Fuzzy Sets}

The Geometric Method avoids type-reduction altogether [4, page 452] “... instead favouring a geometric interpretation of the centre of the FOU of the consequent 
set." The FOU is already a closed polygon to which the same formula that is used for type-1 Geometric Defuzzification is applied [2, page 83].

Definition 19 (Centroid of a Geometric Interval Type-2 Fuzzy Set). "Let Ã be a non-empty geometric interval type-2 fuzzy set whose FOU is in the form of a closed non-intersecting polygon, consisting of $n$ vertices $\left(x_{1}, y_{1}\right),\left(x_{2}, y_{2}\right), \ldots$, $\left(x_{n}, y_{n}\right)$. The centroid of $\tilde{A}$ is calculated by this formula:

$$
X_{\tilde{A}}=\frac{\sum_{i=1}^{i=n}\left(x_{i}+x_{i+1}\right)\left(x_{i} y_{i+1}-x_{i+1} y_{i}\right)}{3 \sum_{i=1}^{i=n}\left(x_{i} y_{i+1}-x_{i+1} y_{i}\right)} .,
$$

\subsection{Geometric Defuzzification of Generalised Type-2 Fuzzy Sets}

For generalised type-2 fuzzy sets the interval Geometric Defuzzification strategy is extended from the FOU to the polyhedron enclosing the membership function [6, page 937], [7, page 94].

Definition 20 (Centroid of a Geometric Generalised Type-2 Fuzzy Set [6, 7]). "Let Ã be a non-empty geometric generalised type-2 fuzzy set whose membership function is in the form of a polyhedron made up of q triangles, whereby the ith triangle $t^{i}$ has vertices $\left(x_{1}^{i}, y_{1}^{i}, z_{1}^{i}\right)$, the $x$-value of the centroid of $t^{i}=C^{i}$, and the area of $t^{i}=A^{i}$. The centroid of $\tilde{A}$ is calculated by these equations:

$$
\begin{aligned}
& C_{\tilde{A}}=\frac{\sum_{i=1}^{i=q} C^{i} A^{i}}{\sum_{i=1}^{i=q} A^{i}} \\
& C^{i}=\frac{x_{1}^{i}+x_{2}^{i}+x_{3}^{i}}{3}, \text { and } \\
& A^{i}=\frac{1}{2} \sqrt{\begin{array}{l}
\left(\left(y_{2}^{i}-y_{1}^{i}\right)\left(z_{3}^{i}-z_{1}^{i}\right)-\left(y_{3}^{i}-y_{1}^{i}\right)\left(z_{2}^{i}-z_{1}^{i}\right)\right)^{2}+ \\
\left(\left(x_{2}^{i}-x_{1}^{i}\right)\left(z_{3}^{i}-z_{1}^{i}\right)-\left(x_{3}^{i}-x_{1}^{i}\right)\left(z_{2}^{i}-z_{1}^{i}\right)\right)^{2}+ \\
\left(\left(x_{2}^{i}-x_{1}^{i}\right)\left(y_{3}^{i}-y_{1}^{i}\right)-\left(x_{3}^{i}-x_{1}^{i}\right)\left(y_{2}^{i}-y_{1}^{i}\right)\right)^{2} .
\end{array}}
\end{aligned}
$$

The next section reports on experiments assessing the accuracy of Geometric Defuzzification of interval type-2 fuzzy sets. 


\section{Accuracy Evaluation of Geometric Defuzzification for Interval Sets}

For type-2 fuzzy sets, the attraction of Geometric Defuzzification is that the computationally complex stage of type-reduction is removed, making the algorithm computationally simple and therefore very fast. Defuzzification algorithms, however, must be accurate as well as efficient. Yet the methods' creators acknowledge there to be an accuracy issue with the outputs of type-2 Geometric Defuzzification for sets with rotational symmetry [7, page 95]: "Clearly, sets with rotational symmetry are problematic to geometric type-2 fuzzy logic." and [6, page 940]: "The only known form of a set that is problematic for the Geometric Defuzzifier is one that has rotational symmetry." They see this as a challenge [7, page 95]: "For type-2 systems, the problem of defuzzifying type-2 fuzzy sets with rotational symmetry remains unsolved." Their confidence in the Geometric Method is undispelled, as they suppose that such type-2 fuzzy sets are unlikely to arise in practical applications [6, page 939]:

"In a real-world Mamdani system, it is unlikely that the final output would ever have the property of rotational symmetry. Rules usually only fire to a degree, and the implication operation (assuming a minimum is used) will produce a result that is not rotational [sic] symmetric, even if the consequent set was rotational symmetric. Typically, more than one rule will fire, leading to a number of implied consequent sets being aggregating [sic] prior to defuzzification. It is highly unlikely that this final aggregated set will be rotational symmetric."

They believe, furthermore, that it is possible to prevent the creation of aggregated sets with rotational symmetry:

"The only known form of a set that is problematic for the geometric defuzzifier is one that has rotational symmetry. However, this result can now be taken into account when designing type- 2 membership functions in a practical system, by avoiding membership functions that are rotationally symmetrical." [6, page 940]

It is hard to imagine how sets with rotational symmetry can be avoided, as membership functions based on S-curves and Z-curves are so prevalent in fuzzy inferencing. Even if it were possible to exclude them from the fuzzification and inferencing stages, it is not obvious that this would prevent the aggregated set having rotational symmetry. Were this avoidance strategy indeed valid, its validity would require proof. 
Whether rotationally symmetrical type-2 aggregated sets are unlikely to occur naturally, and whether their occurrence can be prevented is immaterial unless it can be established that it is only rotationally symmetrical type- 2 fuzzy sets that are susceptible to accuracy issues under Geometric Defuzzification. In the following subsections, the scope of the accuracy issue is surveyed experimentally, to establish, in the interval case, what forms of type- 2 fuzzy sets are affected by the accuracy problem. Beginning with sets that have rotational symmetry, interval test sets with a variety of shapes are defuzzified, firstly by means of the benchmark Exhaustive Method, and secondly, using the Geometric Method; the resultant defuzzified values are contrasted (Table 1). Fourteen interval type-2 fuzzy sets are tested altogether. The first ten are specifically constructed to exhibit certain characteristics. The last four are aggregated sets taken from a prototype Type-2 FIS.

\subsection{Interval Sets Having Rotational Symmetry}

In Figure 2, interval Test Set TSY is depicted. This set has rotational symmetry, and is comparable to a problematic example provided by the authors $[7$, page 95]. There is a huge discrepancy between the geometric and exhaustive defuzzified values. Test Set TSZ (Figure 3) is a reflection of TSY in the line $x=0.5$. Exhaustive defuzzification of sets TSY and TSZ gives a range of defuzzified values of 0.432 , whereas counterintuitively, Geometric Defuzzification gives the identical value of 0.500 for both test sets.

\subsection{Interval Sets Not Having Rotational Symmetry}

The test sets featured in this subsection do not have any symmetry. Test Set NSY (Figure 4), which is Test Set TSA slightly modified so that it is not perfectly rotationally symmetrical, nonetheless exhibits a striking discrepancy between its geometric defuzzified value and its exhaustive defuzzified value. Indeed the discrepancy is worse than for Test Set TSA. Test Sets NSP, NSQ, and NSR (Figures 5 to 7) perform badly under geometric defuzzification; for Test Sets NSQ and NSR one might expect better results from random generation of a number between 0 and 1.

\subsection{Interval Sets Having Reflectional Symmetry}

The test sets considered in this subsection all have reflectional symmetry. Test Set HMSA (Figure 8) has horizontal symmetry; the Geometric Defuzzifier performs poorly with this set. Test Sets VMSC and VMST (Figures 9 and 10) have vertical symmetry, VMSC being centrally placed along the domain and VMSC 


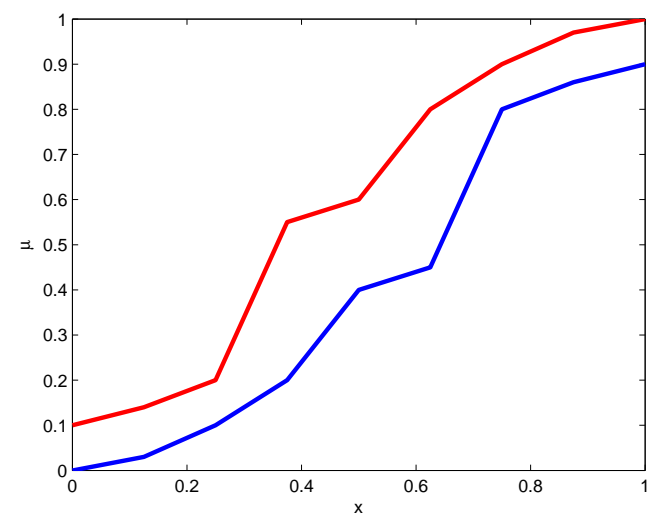

Figure 2: FOU of Test Set TSY, which has rotational symmetry. The degree of discretisation is 0.125. Exhaustive defuzzified value: 0.716. Geometric defuzzified value: 0.500. Discrepancy: 0.216 .

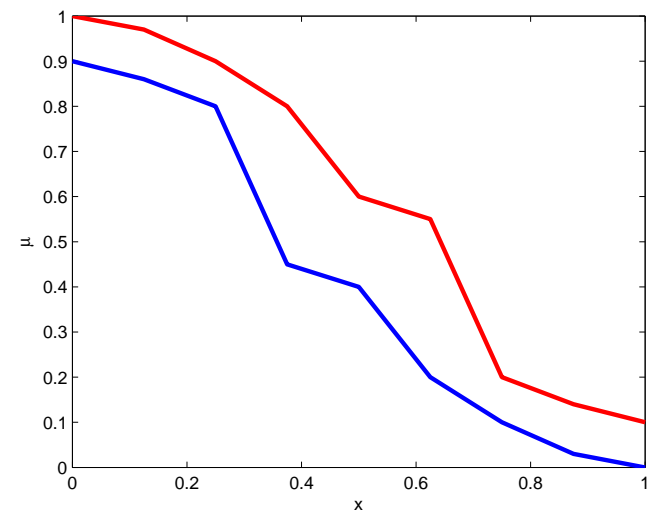

Figure 3: FOU of Test Set TSZ, which has rotational symmetry. The degree of discretisation is 0.125 . Exhaustive defuzzified value: 0.284 . Geometric defuzzified value: 0.500 . Discrepancy: 0.216 . 


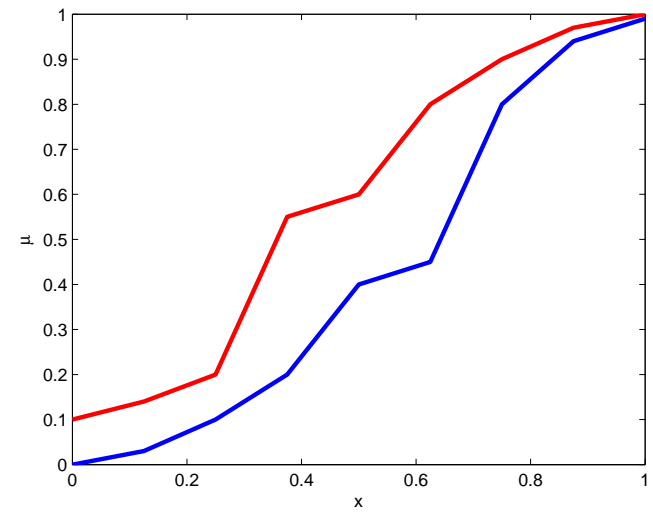

Figure 4: FOU of Test Set NSY, which almost has rotational symmetry. The degree of discretisation is 0.25 . Exhaustive defuzzified value: 0.720 . Geometric defuzzified value: 0.461 . Discrepancy: 0.259 .

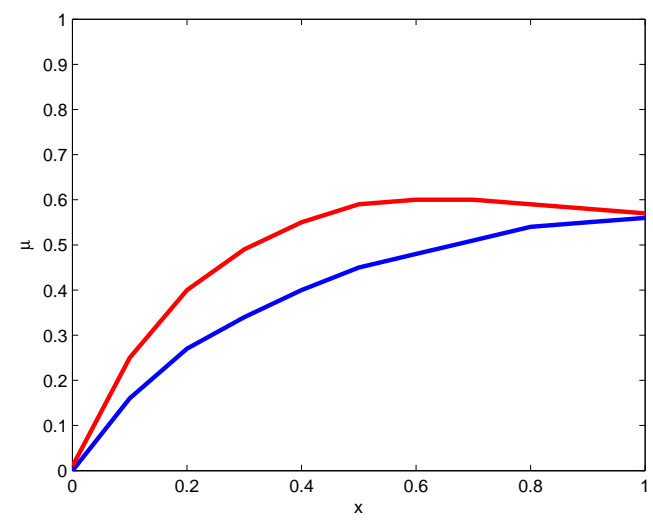

Figure 5: FOU of Test Set NSP, which has no symmetry. The degree of discretisation is 0.1 . Exhaustive defuzzified value: 0.612 . Geometric defuzzified value: 0.434 . Discrepancy: 0.178. 


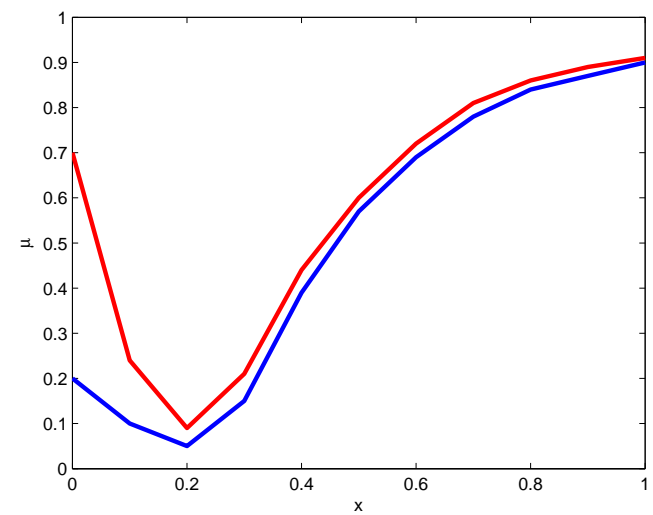

Figure 6: FOU of Test Set NSQ, which has no symmetry. The degree of discretisation is 0.1 . Exhaustive defuzzified value: 0.651 . Geometric defuzzified value: 0.239 . Discrepancy: 0.412 .

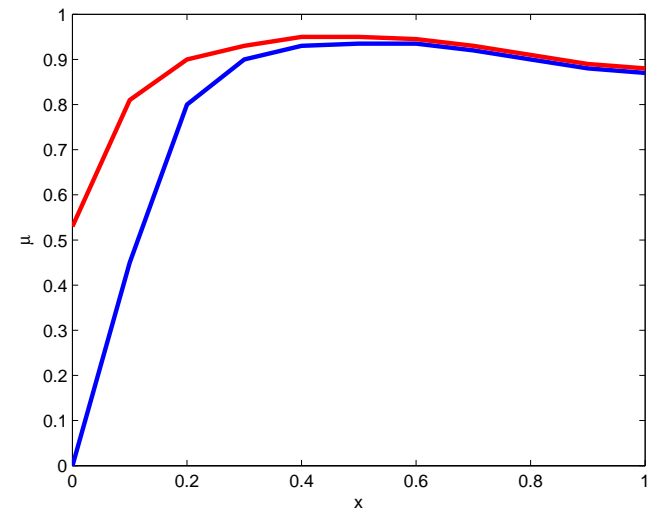

Figure 7: FOU of Test Set NSR, which has no symmetry. The degree of discretisation is 0.1 . Exhaustive defuzzified value: 0.547 . Geometric defuzzified value: 0.149 . Discrepancy: 0.398 . 
placed off-centre. For both these test sets, the Geometric Defuzzifier gives completely precise results. Test Set TMSA (Figure 11) has both rotational and vertical symmetry. Despite its rotational symmetry, with this test set the Geometric Defuzzifier gives totally accurate results.

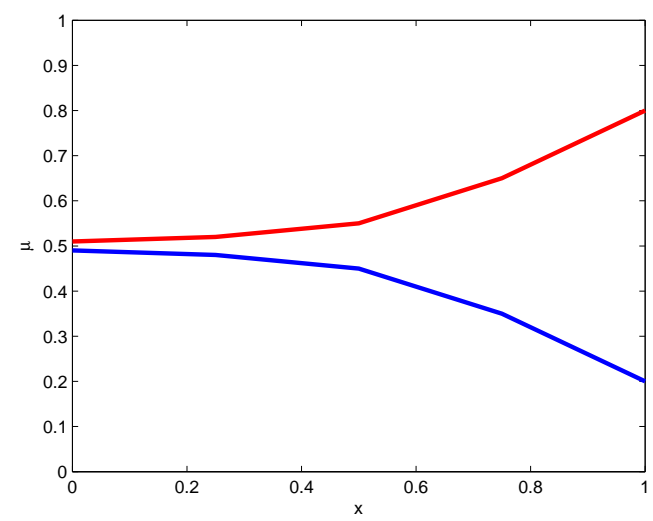

Figure 8: FOU of Test Set HMSA, which has horizontal symmetry. The degree of discretisation is 0.25 . Exhaustive defuzzified value: 0.492 . Geometric defuzzified value: 0.748 . Discrepancy: 0.256 .

\subsection{FIS Generated Aggregated Sets}

It may be objected that the foregoing examples of interval type-2 fuzzy sets are contrived and unrealistic, and therefore that those giving rise to discrepancies are not valuable as counterexamples. To counteract such an objection, this subsection reports trials on four FOUs from aggregated sets (Figure 1) output by the inferencing stage of a prototype type-2 FIS, coded in Matlab ${ }^{T M}$. In effect these trials contrast the performance of the Geometric Method with that of the Exhaustive Method, in the context of an FIS.

Three rule sets were employed ${ }^{2}$ :

Heater FIS, with 5 rules and 2 inputs, is designed to calculate the optimum setting for a heater.

Washing Powder FIS, with 4 rules and 3 inputs, aims to determine the quantity of washing powder required by a washing machine.

\footnotetext{
${ }^{2}$ Specific details of these rule sets may be found in [11].
} 


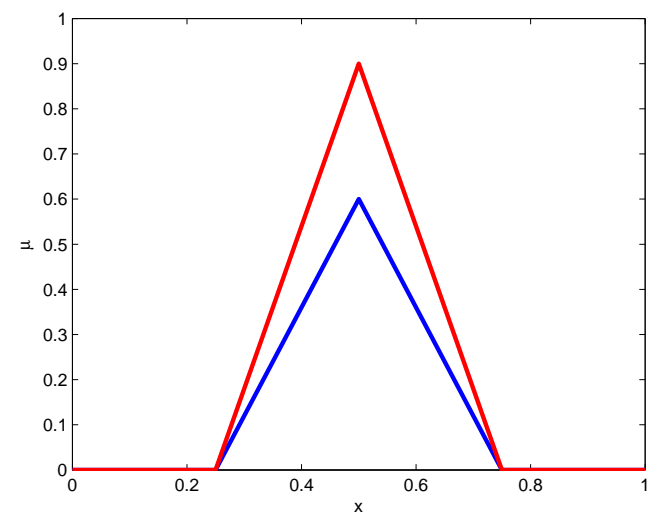

Figure 9: FOU of Test Set VMSC, which has vertical symmetry and is centrally placed along the domain. The degree of discretisation is 0.25 . Exhaustive defuzzified value: 0.500 . Geometric defuzzified value: 0.500 . Discrepancy: 0.000 .

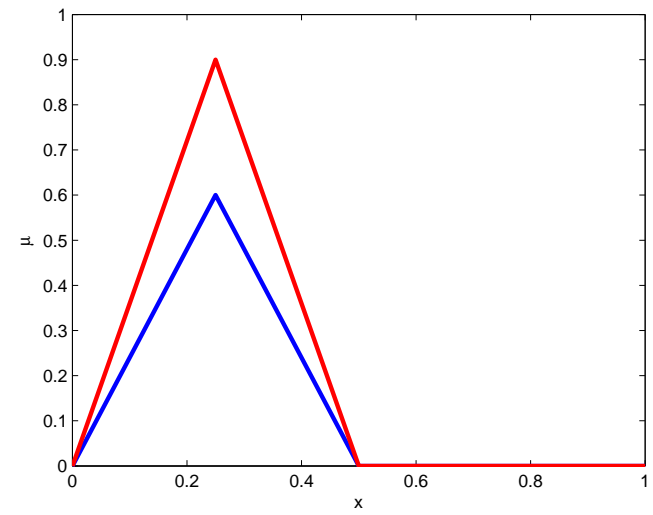

Figure 10: FOU of Test Set VMST, which has vertical symmetry and is not centrally placed along the domain. The degree of discretisation is 0.25 . Exhaustive defuzzified value: 0.250 . Geometric defuzzified value: 0.250 . Discrepancy: 0.000 . 


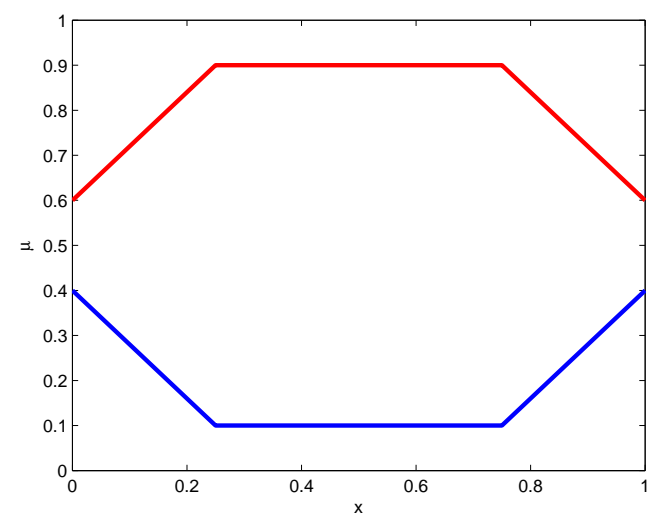

Figure 11: FOU of Test Set TMSA, which has both vertical and horizontal symmetry. The degree of discretisation is 0.25 . Exhaustive defuzzified value: 0.500 . Geometric defuzzified value: 0.500 . Discrepancy: 0.000 .

Shopping FIS, with 4 rules and 3 inputs is intended to answer the dilemma of whether to go shopping by car, or walk, depending on weather conditions, amount of shopping, etc.. The defuzzified value is therefore rounded to either 0 or 1 .

For each rule set the FIS was invoked with two different sets of parameters, so creating six test sets $[9,11]$. Two of these were discarded as they possess near perfect rotational symmetry and such sets have already been discussed (Subsection 4.1). This left four test sets, two for the Heater FIS, and one each from the Washing Powder FIS and the Shopping FIS ${ }^{3}$.

The aggregated type-2 fuzzy sets created through inferencing were converted from generalised type-2 fuzzy sets to interval type- 2 fuzzy test sets, simply by changing all secondary membership grades to 1 . These test sets are depicted in Figures 12 to 15 . All four defuzzification tests show wide discrepancies between the exhaustive defuzzified value and the geometric defuzzified value. The closest result is for Test Set HeaterTest0.125; unsurprisingly this set has some semblance of vertical symmetry (Figure 12).

\footnotetext{
${ }^{3}$ Heater0p0625 is not a more finely discretised version of Heater0p125; it uses different input parameters.
} 


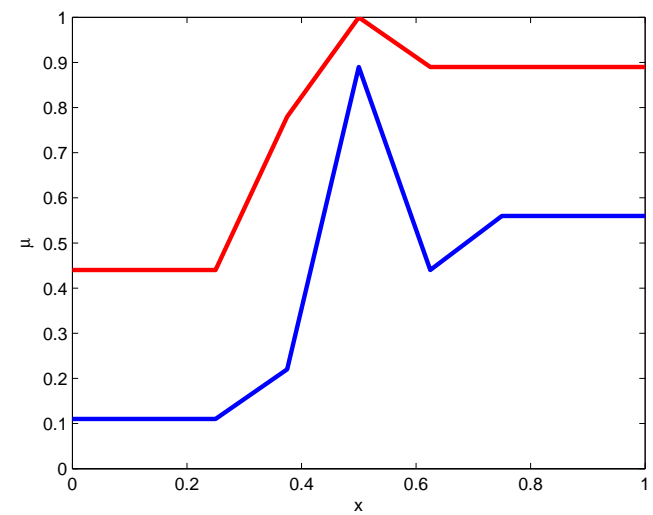

Figure 12: FOU of Test Set HeaterTest 0.125 . The degree of discretisation is 0.125 . Exhaustive defuzzified value: 0.604. Geometric defuzzified value: 0.495. Discrepancy: 0.109.

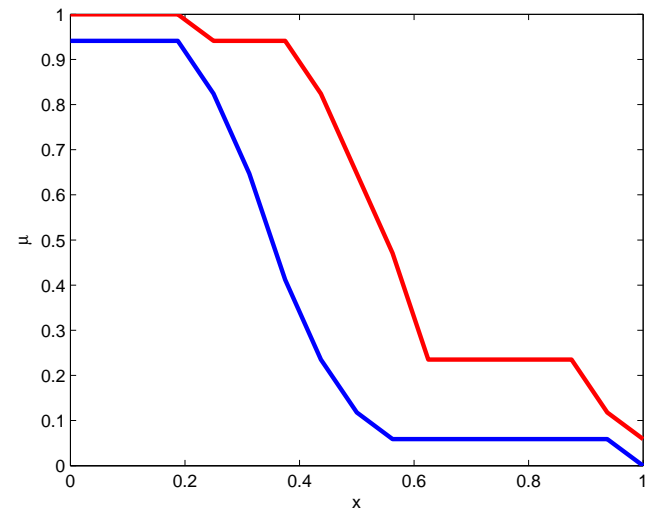

Figure 13: FOU of Test Set HeaterTest0.0625. The degree of discretisation is 0.0625. Exhaustive defuzzified value: 0.280 . Geometric defuzzified value: 0.505 . Discrepancy: 0.225. 


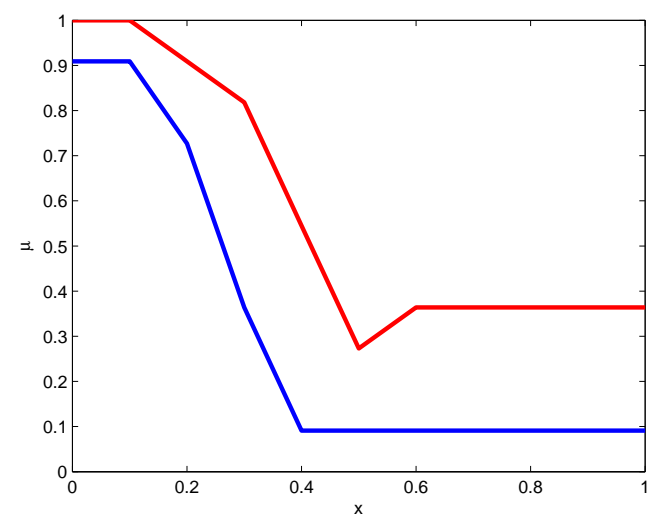

Figure 14: FOU of Test Set powderTest0.1. The degree of discretisation is 0.1. Exhaustive defuzzified value: 0.313 . Geometric defuzzified value: 0.533 . Discrepancy: 0.220.

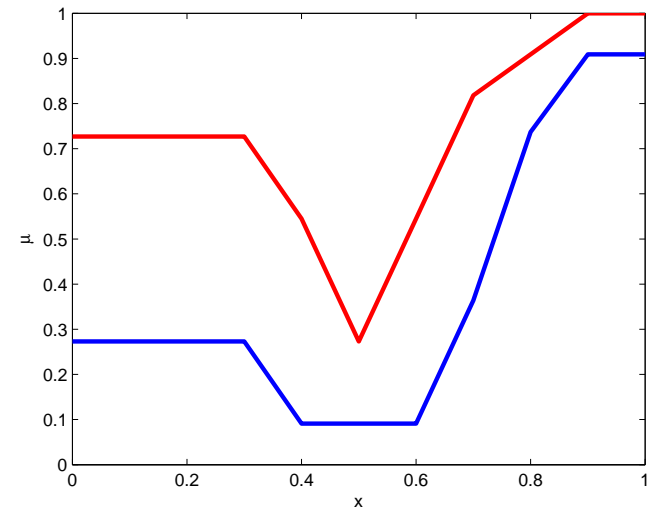

Figure 15: FOU of Test Set shoppingTest0.1. The degree of discretisation is 0.1. Exhaustive defuzzified value: 0.589 . Geometric defuzzified value: 0.409. Discrepancy: 0.180. 


\subsection{Summary of Interval Results}

For the fourteen test sets, Table 1 summarises the results contrasting the exhaustive interval type-2 defuzzified value with the geometric interval type- 2 defuzzified value. Apart from the test sets with vertical symmetry, the results are so poor that there is not a single instance in which even the first decimal place of the defuzzified value is correct.

\begin{tabular}{|l|l|l|l|}
\hline $\begin{array}{l}\text { Test } \\
\text { Set }\end{array}$ & $\begin{array}{l}\text { Exhaustive } \\
\text { Defuzzified } \\
\text { Value }\end{array}$ & $\begin{array}{l}\text { Geometric } \\
\text { Defuzzified } \\
\text { Value }\end{array}$ & $\begin{array}{l}\text { Discre- } \\
\text { pancy }\end{array}$ \\
\hline \hline TSY & 0.716 & 0.500 & 0.216 \\
TSZ & 0.284 & 0.500 & 0.216 \\
\hline NSY & 0.720 & 0.461 & 0.259 \\
NSP & 0.612 & 0.434 & 0.178 \\
NSQ & 0.651 & 0.239 & 0.412 \\
NSR & 0.547 & 0.149 & 0.398 \\
\hline HMSA & 0.492 & 0.748 & 0.256 \\
VMSC & $\mathbf{0 . 5 0 0}$ & $\mathbf{0 . 5 0 0}$ & $\mathbf{0 . 0 0 0}$ \\
VMST & $\mathbf{0 . 2 5 0}$ & $\mathbf{0 . 2 5 0}$ & $\mathbf{0 . 0 0 0}$ \\
TMSA & $\mathbf{0 . 5 0 0}$ & $\mathbf{0 . 5 0 0}$ & $\mathbf{0 . 0 0 0}$ \\
\hline HeaterTest 0.125 & 0.604 & 0.495 & 0.109 \\
HeaterTest 0.0625 & 0.280 & 0.505 & 0.225 \\
PowderTest 0.1 & 0.313 & 0.533 & 0.220 \\
ShoppingTest 0.1 & 0.589 & 0.409 & 0.180 \\
\hline \hline
\end{tabular}

Table 1: The test results for fourteen interval type-2 fuzzy test sets contrasting the exhaustive defuzzified value with the geometric defuzzified value. The results for the three test sets with vertical symmetry are shown in bold.

In the next section, Geometric Defuzzification is critiqued theoretically, exposing the reason for the experimental discrepancies observed in this section.

\section{Theoretical Critique of Geometric Type-2 Defuzzification}

In this section, the issue of the accuracy of type-2 Geometric Defuzzification is examined theoretically. For the majority of the fourteen defuzzified test sets (Subsections 4.1 to 4.4), the Geometric Defuzzifier's outputs are wildly inaccurate. 
The exceptions are those sets which have vertical symmetry, for which Geometric Defuzzification gives absolutely accurate results.

How can these astonishing results be explained? In extending defuzzification from type- 1 to interval type- 2 fuzzy sets, the $x$-value of the centroid of the area under the membership function curve still needs to be calculated, but, the membership function having been blurred, this is no longer straightforward.

For discretised interval type-2 fuzzy sets, the Exhaustive Method of Defuzzification [12] is rigourous and precise, but impractical owing to its enormous computational complexity. Three practical alternatives are the Nie-Tan Method [25], the Greenfield-Chiclana Collapsing Defuzzifier [16], and the Karnik-Mendel Iterative Procedure [20]. These techniques are all good approximations to Exhaustive Defuzzification [12], and all involve some form of type-reduction. In the case of the Nie-Tan Method the resultant type-1 fuzzy set consists of the midpoints of the LMF and the UMF. For the Collapsing Defuzzifier, the resultant type- 1 set approximates to that of the Nie-Tan Method ${ }^{4}$. As well as providing a good approximation to the defuzzified value, defuzzifying the type- 1 set formed from the midpoints of the LMF and the UMF is an intuitively appropriate ad hoc procedure.

The Geometric Defuzzifier finds the $x$-value of the centroid of the area between the LMF and the UMF of the blurred membership function curve. I.e. it defuzzifies the FOU of the type-2 set rather than the blurred membership function. If a fuzzy set's membership function is rotated, one would expect its defuzzified value to change. Under type-2 Geometric Defuzzification, it remains the same.

The experimental discrepancies are attributable to the Geometric Defuzzifier's misapplication of the centroid formula. The authors claim that

"Geometric defuzzification finds the center of the area of the membership function of the type-2 fuzzy set." [6, page 938]

However they are mistaken; this is what is required, but not what is achieved. Their interval approach is misguided as they apply the centroid formula to the FOU itself rather than the membership function.

Their extension of their interval method to generalised type-2 fuzzy sets suffers from the same fallacy as their interval approach since it is a 3-D version of the same specious technique. It is intrinsically unreliable. In [6, pages 938-939] the authors describe testing type- 2 Geometric Defuzzification on three generalised

\footnotetext{
${ }^{4}$ The Karnik-Mendel Iterative Procedure type-reduces to the TRS in a manner entirely different from that of the Nie-Tan and Collapsing Methods.
} 
test sets, one with perfect vertical symmetry, one close to having vertical symmetry, and one based on an S-curve. Naturally the first test set performed perfectly with the Geometric Defuzzifier, and the second performed well. The third test set performed poorly, which reinforced the spurious supposition that only sets with rotational symmetry cause problems for type-2 Geometric Defuzzification. Perhaps if further trials had been undertaken on a range of test sets with more varied characteristics (as in [11] and [12]) before testing on a mobile robot navigation FIS $[2,8]$, a fuller picture of the extent of the method's shortcomings would have emerged.

Consider an interval type-2 fuzzy set that has vertical symmetry in the line $x=a$. Its FOU has vertical symmetry in its line of symmetry $(x=a)[1$, page 668]. The defuzzified value of a type-2 fuzzy set that has vertical symmetry in the line $x=a$ is $a$. This is because the TRS is formed from a collection of embedded sets, each of which has its reflection in the line $x=a$. The defuzzified values of any embedded set and its reflected counterpart will be equidistant to but on opposite sides of the line $x=a$; the TRS interval will therefore defuzzify to $x=a$.

This explains why the interval type-2 Geometric Defuzzifier gives accurate results for type- 2 fuzzy sets which have vertical symmetry. These sets form a special group for which the centre of area (or volume) of the FOU (or volume under the surface of the membership function) is equal to the defuzzified value of the blurred membership function. The closer a set approximates to vertical symmetry, the more accurate will be the results of the technique ${ }^{5}$. It is the lack of vertical symmetry, not the presence of rotational symmetry in a type-2 fuzzy set, that determines whether Geometric Defuzzification will give rise to an accurate defuzzified value. If a fuzzy set has vertical symmetry, then Geometric Defuzzification will give perfect results, even if the set also has rotational symmetry, as in the case with Test Set TMSA (Figure 11).

In their mistaken belief that only rotationally symmetrical type- 2 fuzzy sets are susceptible to accuracy issues under Geometric Defuzzification, the methods' creators offer this explanation for the problems [7, pages 94-95]:

"The membership function of a geometric type-1 fuzzy set is a piecewiselinear function, which is closed to form a polygon for defuzzification.

For a geometric type-2 fuzzy set the membership function is already a closed polyhedron. So, why is this important? It means a type-

\footnotetext{
${ }^{5}$ To explain the success of the testing on mobile robots one might speculate that the FOUs of the type-2 fuzzy sets approximated to vertical symmetry.
} 
1 geometric membership function can only ever have on [sic] line of symmetry parallel to the $y$ axis. A type-2 geometric membership function can also have a line [sic] of rotational symmetry. A geometric defuzzifier will identify this line of symmetry, which may not be the required answer."

Both type- 1 and type- 2 fuzzy sets may have rotationally symmetrical membership functions. The authors' argument appears to be that for a type-2 fuzzy set with rotational symmetry, the Geometric Defuzzifier will identify the point about which the blurred region (i.e. the FOU in the interval case, the volume under the surface of the membership function in the generalised case), is rotationally symmetrical. Perhaps unwittingly, they actually point out the intrinsic fallacy of their method: Rather than defuzzifying the membership function, the type-2 Geometric Defuzzifier finds the centroid of the blurred region within the type- 2 fuzzy membership function.

\section{Conclusions}

1. Far from suffering from a peculiarity restricted to type-2 sets with rotational symmetry, the Geometric Defuzzifier in its application to type-2 fuzzy sets is deeply flawed, as evidenced by the numerous examples presented of wildly inaccurate performance on test sets. Indeed the only situation in which the Geometric Defuzzifier may be relied upon is where the type-2 fuzzy set has vertical symmetry, for which the centroid is easily found as it lies on the line of symmetry. Contrary to its developers' intentions (Section 1), type-2 Geometric Defuzzification is by no means "...precise and accurate so that correct decisions are taken.”[6, page 929]

2. The unexpected and unacceptable results have been shown to derive from fallacious reasoning. In the interval case it is wrongly assumed that finding the centroid of the FOU is equivalent to defuzzifying the blurred membership function of the interval type-2 fuzzy set. In the generalised case it is wrongly assumed that finding the centroid of the volume under the surface of the membership function is equivalent to defuzzifying the blurred membership function of the generalised type-2 fuzzy set.

3. Since it is demonstrably not fit for purpose, the type-2 Geometric Defuzzifier is best avoided by developers of Type-2 FIS applications. 
4. In order to quickly identify accuracy issues in proposed defuzzification algorithms, it is prudent to test them using a varied range of test sets before evaluating them in the context of an FIS.

5. If possible, it is advisable to formally prove the correctness of defuzzification algorithms mathematically.

\subsection{Further Work}

\subsubsection{Distinction between Discretised and Geometric Fuzzy Sets}

Is the distinction between geometric fuzzy sets and discretised fuzzy sets genuine, or merely one of presentation? Both forms of fuzzy sets are defined as a collection of points (Subsections 1.1.1 and 1.1.2). As shown in Section 4, there is no difficulty in creating type- 1 and interval type- 2 test sets that may be construed as either geometric or discretised.

\subsubsection{Non-Geometric Type-2 Fuzzy Inferencing Applications}

The authors of the Geometric Defuzzifier developed a mobile generalised type-2 robot navigation FIS [2, 8], which performed well, outperforming both type-1 and interval type-2 fuzzy logic controllers on the same task [8]. Since, for type-2 fuzzy sets, the Geometric Defuzzifier outperformed type-1 fuzzy inferencing even with a flawed approach to defuzzification, using a sound defuzzifier is likely to give even better results. More applications need to be developed using defuzzification techniques that have stood up well to testing on test sets, such as the interval Nie-Tan Method [25] and Greenfield-Chiclana Collapsing Defuzzifier [16], both evaluated in [12], and the generalised sampling method [15, 19] and Vertical Slice Centroid Type-Reduction [22], both evaluated in [11].

\section{References}

[1] L. Bostock and S. Chandler. Pure Mathematics I. Stanley Thornes, 1978.

[2] S. Coupland. Geometric Fuzzy Logic Systems. PhD thesis, De Montfort University, 2006.

[3] S. Coupland. Type-2 Fuzzy Sets: Geometric Defuzzification and TypeReduction. In Proc. FOCI 2007, pages 622 - 629, Honolulu, Hawaii, USA, April 2007. 
[4] S. Coupland and R. I. John. Geometric Interval Type-2 Fuzzy Systems. In Proceedings of EUSFLAT-LFA 2005, pages 449 - 454, Barcelona, Spain, September 2005.

[5] S. Coupland and R. I. John. Geometric Type-1 and Type-2 Fuzzy Logic Systems. IEEE Transactions on Fuzzy Systems, 15(1):3 - 15, February 2007.

[6] S. Coupland and R. I. John. A fast geometric method for defuzzification of type-2 fuzzy sets. IEEE Transactions on Fuzzy Systems, 16(4):929 - 941, August 2008.

[7] S. Coupland and R. I. John. Geometric type-2 fuzzy sets. In A. Sadeghian, J. M. Mendel, and H. Tahayori, editors, Advances in Type-2 Fuzzy Sets and Systems, volume 301 of Studies in Fuzziness and Soft Computing, pages 81 - 96. Springer New York, January 2013. ISBN 978-1-4614-6665-9.

[8] S. Coupland, M. Gongora, R. I. John, and K. Wills. A comparative study of fuzzy logic controllers for autonomous robots. In Proc. IPMU 2006, pages 1332 - 1339, Paris, July 2006.

[9] S. Greenfield. PhD thesis, De Montfort University, 2012. URL: http://hdl. $\mathrm{PhD}$ thesis, De Montfort University, 2012.

[10] S. Greenfield and F. Chiclana. Combining the $\alpha$-Plane Representation with an Interval Defuzzification Method. In Proceedings of EUSFLAT-LFA 2011, pages 920 - 927, Aix-les-Bains, France, July 2011.

[11] S. Greenfield and F. Chiclana. Defuzzification of the Discretised Generalised Type-2 Fuzzy Set: Experimental Evaluation. Information Sciences, 244:1 25, September 2013. DOI: http://dx.doi.org/10.1016/j.ins.2013.04.032.

[12] S. Greenfield and F. Chiclana. Accuracy and Complexity Evaluation of Defuzzification Strategies for The Discretised Interval Type-2 Fuzzy Set. International Journal of Approximate Reasoning, 54(8):1013 - 1033, October 2013. DOI: http://dx.doi.org/10.1016/j.ijar.2013.04.013.

[13] S. Greenfield and F. Chiclana. Fuzzy in 3D: Two Contrasting Paradigms. Archives for the Philosophy and History of Soft Computing, 2, 2015. http://www.aphsc.org/index.php/aphsc/article/view/24 [Online; accessed 20-January-2016]. 
[14] S. Greenfield and F. Chiclana. Slicing Strategies for the Generalised Type-2 Mamdani Fuzzy Inferencing System. pages 195 - 205, Zakopane, Poland, 2016. 15th International Conference on Artificial Intelligence and Soft Computing (ICAISC 2016).

[15] S. Greenfield, R. I. John, and S. Coupland. A Novel Sampling Method for Type-2 Defuzzification. In Proc. UK Workshop on Computational Intelligence 2005, pages 120 - 127, London, September 2005.

[16] S. Greenfield, F. Chiclana, S. Coupland, and R. I. John. The Collapsing Method of Defuzzification for Discretised Interval Type-2 Fuzzy Sets. Information Sciences, 179(13):2055 - 2069, June 2009. DOI: http://dx.doi.org/10.1016/j.ins.2008.07.011.

[17] S. Greenfield, F. Chiclana, and R. I. John. Type-Reduction of the Discretised Interval Type-2 Fuzzy Set. In Proceedings of FUZZ-IEEE 2009, pages 738 - 743, Jeju Island, Korea, August 2009.

[18] S. Greenfield, F. Chiclana, S. Coupland, and R. I. John. Type2 Defuzzification: Two Contrasting Approaches. In Proceedings of FUZZ-IEEE 2010, pages 1 - 7, Barcelona, July 2010. DOI: http://dx.doi.org/10.1109/FUZZY.2010.5584007.

[19] S. Greenfield, F. Chiclana, R. I. John, and S. Coupland. The Sampling Method of Defuzzification for Type-2 Fuzzy Sets: Experimental Evaluation. Information Sciences, 189:77 - 92, April 2012. DOI: http://dx.doi.org/10.1016/j.ins.2011.11.042.

[20] N. N. Karnik and J. M. Mendel. Centroid of a Type-2 Fuzzy Set. Information Sciences, 132:195 - 220, 2001.

[21] W. Van Leekwijck and E. E. Kerre. Defuzzification: Criteria and Classification. Fuzzy Sets and Systems, 108:159 - 178, 1999. DOI: 10.1016/j.fss.2008.06.018.

[22] L. Alberto Lucas, T. Mezzadri Centeno, and M. Regattieri Delgado. General Type-2 Fuzzy Inference Systems: Analysis, Design and Computational Aspects. In Proceedings of FUZZ-IEEE 2007, pages 1743-1747, London, 2007. 
[23] J. M. Mendel. Uncertain Rule-Based Fuzzy Logic Systems: Introduction and New Directions. Prentice-Hall PTR, 2001. ISBN 0-13-040969-3.

[24] J. M. Mendel and R. I. John. Type-2 fuzzy sets made simple. IEEE Transactions on Fuzzy Systems, 10(2):117 - 127, 2002. DOI: http://dx.doi.org/10.1109/91.995115.

[25] M. Nie and W. W. Tan. Towards an Efficient Type-Reduction Method for Interval Type-2 Fuzzy Logic Systems. In Proceedings of FUZZ-IEEE 2008, pages 1425 - 1432, Hong Kong, June 2008.

[26] L. A. Zadeh. Fuzzy Sets. Information and Control, 8:338 - 353, 1965.

[27] L. A. Zadeh. The Concept of a Linguistic Variable and its Application to Approximate Reasoning. Information Sciences, 8:199 - 249, 1975.

[28] L. A. Zadeh. The Concept of a Linguistic Variable and its Application to Approximate Reasoning - II. Information Sciences, 8:301 - 357, 1975.

[29] L. A. Zadeh. The Concept of a Linguistic Variable and its Application to Approximate Reasoning - III. Information Sciences, 9:43 - 80, 1975. 